\title{
Effect of antifungal treatment on the prevalence of yeasts in HIV-infected subjects
}

Correspondence

Mrudula Patel

patelms@dentistry.wits.ac.za

Received 23 February 2006

Accepted 17 May 2006
Mrudula Patel, Joanne T. Shackleton and Maeve M. Coogan

Division of Oral Microbiology, Department of Clinical Microbiology and Infectious Diseases, Faculty of Health Sciences and University of the Witwatersrand, Johannesburg, South Africa

Oral candidiasis, the most common opportunistic infection in patients with HIV infection, is usually associated with Candida albicans. Several factors may influence the carriage of Candida, including immunocompromised conditions and HIV infection, colonization by yeasts from different geographical areas and antimycotic treatment. This study investigated the Candida carrier rate, level and types of yeast in HIV-positive and -negative subjects, and the effect of previous exposure to antifungal drugs on the level of yeasts in HIV-positive patients in Gauteng, South Africa. Unstimulated saliva was collected from 332 HIV-positive patients and 100 HIV-negative subjects and cultured for yeasts. The number and species of yeast were determined. HIV-positive patients who carried yeasts were divided into two groups depending upon their previous antifungal drug exposure, and the level of Candida carriage in each group was compared. The Candida carrier rate in the HIV-positive patients $(81 \cdot 3 \%)$ was slightly higher than previously reported and significantly higher $(P<0.001)$ than in the HIV-negative group (63\%). The carrier rate in the HIV-negative group was also higher than in earlier studies. Fourteen per cent of the HIV-positive patients carried more than 10000 c.f.u. $\mathrm{ml}^{-1}$ whereas none of the HIV-negative subjects carried this large a number of yeasts $(P<0.001)$. Seventy per cent of the yeasts were identified as C. albicans and approximately $30 \%$ as non-albicans species. In conclusion, the Candida carrier rate is higher in the South African population than elsewhere. HIV-positive patients carry more and a greater variety of yeasts than HIV-negative subjects. Exposure to antifungal drugs has no effect on the level of yeast carriage in HIV-positive patients.

\section{INTRODUCTION}

Oral candidiasis is the most frequently encountered opportunistic infection in patients with HIV and AIDS (Patton et al., 2002). Several studies have shown that asymptomatic carriage of oral Candida species varies geographically in healthy and compromised individuals (Table 1). Reports vary in different regions of South Africa. In the Western Cape, a province of South Africa, the rate was higher, with $68 \%$ of HIV-negative subjects and $75 \%$ of HIV-positive patients carrying yeasts (Hauman et al., 1993). In this paper, only 28 HIV-positive patients were investigated, while the level of carriage was unclear. Most of the yeasts were identified as Candida albicans. In patients whose HIV status was unknown, a rate of $30.4 \%$ was reported in the Kalahari in a rural area of South Africa and $58 \%$ in the semiurban region of Gauteng province (Blignaut et al., 1995; Masipa et al., 1992).

C. albicans is the most common species of yeast isolated from patients with oral candidiasis (Fidel, 2006). There are several reports that non-albicans yeasts including Candida glabrata, Candida krusei, Candida parapsilosis, Candida tropicalis, Candida guilliermondii, Candida dubliniensis and Saccharomyces cerevisiae have been isolated from the oral cavity of immunocompromised patients (Powderly, 1992; Pfaller, 1996). Recent studies have shown that non-albicans species may become pathogenic in HIV-negative patients. For example, C. glabrata, C. parapsilosis, C. tropicalis and S. cerevisiae have been isolated from infections in neonates, secondary sepsis in bone marrow transplant patients, and fungaemia (Roilides et al., 2003; Cherifi et al., 2004; Henry et al., 2004; Redding et al., 2004a). This information may change the perception of non-albicans yeasts from opportunistic to exogenous infective agents that can be transmitted readily from person to person and be easily acquired by immunocompromised people (Pfaller, 1996; Sanchez et al., 1993; Doebbeling et al., 1991).

Candida carriage and candidiasis may also be affected by several factors including HIV infection (Diamond, 1991), immunosuppressive drug therapy, cytotoxic therapy, intensive care treatment, iron status of the host and the prolonged use of antibiotics (Bodey et al., 1992; Paya, 1993; Pfaller, 1996; Blignaut et al., 1995; Pendrak et al., 2004). The emergence of less common yeasts could be caused by the selection of resistant species by the pressure of antifungal agents such as fluconazole (Price et al., 1994; Wingard et al., 1991; Wingard, 1994). 
Table 1. Reports of Candida carrier rate in HIV-positive and HIV-negative subjects in Asia, Europe and Africa

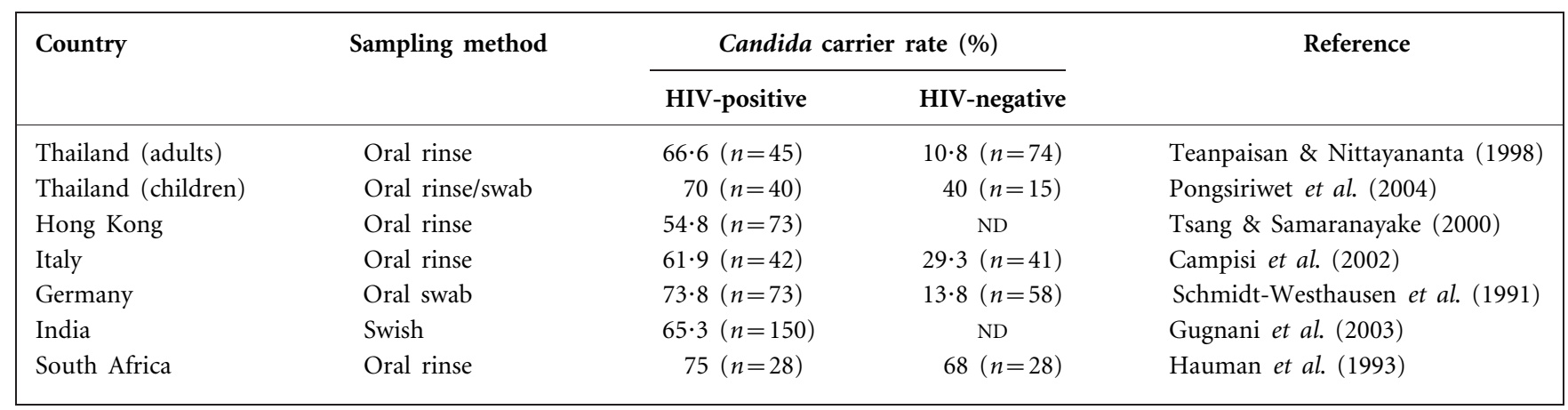

ND, Not determined.

The aim of this study was to compare the prevalence and level of yeast species in HIV-positive patients and HIVnegative subjects in South Africa. In addition the effect of previous antifungal treatment on the carrier rate of yeasts in HIV-positive patients was investigated.

\section{METHODS}

Subjects and collection of saliva. The study population was selected from patients attending the HIV clinic at the New Johannesburg and Chris Hani Baragwanath Hospitals, Gauteng, a single geographical locale. The HIV-negative patients were healthy individuals who volunteered to participate in an ongoing HIV vaccine trial at Chris Hani Baragwanath Hospital and were screened for their HIV status. The first $332 \mathrm{HIV}$-positive and $100 \mathrm{HIV}$-negative subjects who volunteered were included in the study regardless of their gender. The population consisted of $332 \mathrm{HIV}$-positive patients ( 88 males with a mean age of $38 \cdot 7$ years, range $21-62$; 244 females mean age 34.9 years, range $16-57)$ and $100 \mathrm{HIV}$-negative subjects ( 55 males with a mean age of $27 \cdot 2$ years, range $18-59 ; 45$ females mean age $26 \cdot 1$, range $18-41$ ). Ethical clearance was obtained from The Committee for Research on Human Subjects, University of the Witwatersrand. Written consent was obtained from the subjects. Ten minute unstimulated saliva samples were collected, cultured on Sabouraud dextrose agar and incubated at $37^{\circ} \mathrm{C}$ for $48 \mathrm{~h}$. Yeast counts were determined by colony morphology and the Gram stain. Previous treatment with antifungal agents was recorded for 255 HIV-positive patients. Of these patients, 177 had never been exposed to antifungal agents while 78 had been treated with either fluconazole, nystatin, myconazole or amphotericin B within the last year.
Amphotericin B lozengers and miconazole gel were applied topically 10 times a day for 1 week while nystatin and fluconazole were used systemically for the same period.

Yeast identification. Isolates from $150 \mathrm{HIV}$-positive patients carrying $\geqslant 1000$ c.f.u. ( $\mathrm{ml}$ saliva) $)^{-1}$ and 63 HIV-negative patients were identified to species level using the germ tube technique, chlamydospore production and the API $32 \mathrm{C}$ sugar assimilation tests (bioMèrieux). Four colonies with different colony morphology per patient were identified.

Germ tube and chlamydospore formation, $\beta$-glucosidase activity and growth at $45^{\circ} \mathrm{C}$ were used to differentiate between $C$. albicans and $C$. dubliniensis (Staib \& Morschhauser, 1999; Pinjon et al., 1998; Boerlin et al., 1995). Counts of individual species were not performed.

Statistical analysis. The yeast carrier rate among HIV-positive and HIV-negative groups and the number of yeast in both study groups were compared using the chi-square test. In addition the chi-square test was used to compare Candida counts in HIV-positive patients who had been previously exposed to antifungal agents to Candida counts in patients with no previous exposure to antimycotics.

\section{RESULTS AND DISCUSSION}

\section{Carriage rate}

The Candida carriage rate (Table 2) in the HIV-positive patients $(81 \cdot 3 \%)$ was significantly higher $(P<0 \cdot 001)$ than in the HIV-negative group $(63 \%)$. This carrier rate was higher than in studies from Thailand (adults $66 \cdot 6 \%$ and children $70 \%)$, Hong Kong (54.8\%), Italy (61.9\%),

Table 2. Viable yeast counts in the whole saliva of HIV-positive and HIV-negative patients

Values are the number of patients (\%).

\begin{tabular}{|lccccc|}
\hline \multirow{2}{*}{ Study group } & Carrier rate (\%) & \multicolumn{4}{c|}{ Yeast count $\left(\mathbf{c . f . u . ~} \mathbf{~ m l}^{\mathbf{- 1}}\right.$ ) } \\
\cline { 3 - 5 } & & $\mathbf{0}$ & $\mathbf{1 - 1 0 0 0}$ & $\mathbf{1 0 0 0 - 1 0 0 0 0}$ & $>\mathbf{1 0 0 0 0}$ \\
\hline HIV-positive & $270(81 \cdot 3)^{*}$ & $62(18 \cdot 7)$ & $116(43) \dagger$ & $115(42 \cdot 6)$ & $39(14 \cdot 4) \dagger$ \\
HIV-negative & $63(63)^{*}$ & $37(37)$ & $45(71 \cdot 4) \dagger$ & $18(28 \cdot 6)$ & $0(0) \dagger$ \\
\hline
\end{tabular}

${ }^{*} P<0 \cdot 001$

$\dagger P=0 \cdot 001$ 
Germany (73.8\%) and India (65.3\%) (Teanpaisan \& Nittayananta, 1998; Pongsiriwet et al., 2004; Tsang \& Samaranayake, 2000; Campisi et al., 2002; SchmidtWesthausen et al., 1991; Gugnani et al., 2003).

Several factors may have contributed to this high carrier rate. Diet could be important because a recent study has shown that malnourished HIV-positive children in Nigeria carried more yeasts than a similar population in the United States (Jabra-Rizk et al., 2001). A study in Turkey showed that different populations and age groups carry different levels of yeasts, while the type of diet may affect the frequency of carriage (Kadir et al., 2005). Our subjects were African adults whose diet is usually high in carbohydrates. However, carbohydrate intake varied according to regions. For example people living in the Kalahari region are nomads and their main diet is animal protein and occasionally carbohydrate, while in the Western Cape province and Gauteng the population is urbanized and their carbohydrate intake is usually high (MacKeown \& Faber, 2004). This could explain the differences between results among the South African studies. The carrier rate was $30.4 \%$ in Kalahari, $58 \%$ in the semiurban area of Gauteng and $68 \%$ in the urbanized Western Cape region. In our study the population was urbanized and their carbohydrate intake was probably high. This enhances the proliferation of Candida (Knight \& Fletcher, 1971), adhesion to epithelial cells (Pizzo et al., 2000) and the production of acidic proteases (Samaranayake et al., 1984). Carriage rate could also be related to the method of sample collection (Table 1). For example using oral swabs and unstimulated saliva may not detect as many Candida as using an oral rinse. In our study, even though unstimulated saliva was tested, the carrier rate was high.

The level of carriage in HIV-positive subjects was significantly higher $(P<0.001)$ than in HIV-negative patients (Table 2). These differences may be related to the level of $\mathrm{CD} 4{ }^{+}$cells, because our patients did not have access to HAART and probably had low $\mathrm{CD} 4^{+}$cell counts. This is supported by Fidel (2006), who suggests that Candidaspecific $\mathrm{T}$ cells do not become defective with immunosuppression, but a threshold number of $\mathrm{CD} 4^{+}$cells is required to protect the oral cavity against infection by this normal commensal. Out of the 39 patients who had more than 10000 Candida (ml saliva) ${ }^{-1}$ only 16 patients had oral lesions. Of these 16 patients with oral candidiasis 3 carried other yeasts in addition to C. albicans. The rest of the patients with high Candida counts and oral candidiasis at the time of sampling carried only C. albicans.

This study showed that $63 \%$ of HIV-negative subjects carried Candida (Table 2). This was higher than reports from Europe $(13 \cdot 8-29 \%)$, Thailand $(10 \cdot 8 \%)$ and from a Saudi population (52\%) (Teanpaisan \& Nittayananta, 1998; Pongsiriwet et al., 2004; Campisi et al., 2002; SchmidtWesthausen et al., 1991; Darwazeh et al., 2002). A recent study reported that oral yeast colonization among AIDS household contacts is high (Milan et al., 2001), which suggests that transfer and colonization of strains can occur readily. The transfer may not be related to HIV status but to exposure to AIDS patients with high counts because $63 \%$ of HIV-negative subjects carried Candida and live in South Africa where more than 5.0 million of the population are living with AIDS (Dorrington et al., 2004). The HIVpositive and -negative patients came from the same low socioeconomic densely populated area, where several families may live in one house and general hygiene is poor.

\section{Species isolated}

C. albicans was the most common species isolated in our study and comprised $78.6 \%$ of the isolates whereas $21.4 \%$ were non-albicans species (Table 3 ). The percentage of nonC. albicans was higher than in reports from Thailand $(3 \cdot 4 \%)$ and Germany $(13 \cdot 2 \%)$ (Teanpaisan \& Nittayananta, 1998; Schmidt-Westhausen et al., 1991). Non-symptomatic oral cavity carriage of non-C. albicans is well established (Kadir et al., 2005; Qi et al., 2005; Torres et al., 2003; Gugnani et al., 2003; Reichart et al., 2002; Belazi et al., 2004; Redding et al., 2004a). A recent epidemiological trend is the emergence of less pathogenic species of non-albicans Candida as significant opportunistic pathogens. Species isolated in our study included C. glabrata, C. tropicalis, C. krusei, C. parapsilosis, $S$. cerevisiae and the newly characterized species C. dubliniensis (Table 3). C. glabrata sepsis has been observed in bone marrow transplant patients and oropharyngeal candidiasis in head and neck cancer patients (Redding et al., 2004a, b). Fungaemia due to C. parapsilosis, C. tropicalis and C. glabrata has been reported in neonates (Roilides et al., 2003) whereas oral candidiasis due to $C$. glabrata has been reported (Hoegl et al., 1998). S. cerevisiae

Table 3. Species of yeast isolated from 150 HIV-positive and 63 HIV-negative patients

\begin{tabular}{|lcc|}
\hline \multirow{2}{*}{ Yeast isolate } & \multicolumn{2}{c|}{ No. of isolates (\%) } \\
\cline { 2 - 3 } & HIV-positive & HIV-negative \\
\hline C. albicans & $136(78 \cdot 6)$ & $45(70 \cdot 3)$ \\
C. dubliniensis & $11(6 \cdot 3)$ & $3(4 \cdot 7)$ \\
C. glabrata & $9(5 \cdot 2)$ & 0 \\
C. parapsilosis & $3(1 \cdot 7)$ & $1(1 \cdot 6)$ \\
C. lusitaniae & $3(1 \cdot 7)$ & 0 \\
S. cerevisiae & $3(1 \cdot 7)$ & $1(1 \cdot 6)$ \\
C. sake & $2(1 \cdot 2)$ & $3(4 \cdot 7)$ \\
C. tropicalis & $1(0 \cdot 6)$ & $2(3 \cdot 1)$ \\
C. krusei & $1(0 \cdot 6)$ & 0 \\
C. famata & $1(0 \cdot 6)$ & 0 \\
C. pelliculosa & $1(0 \cdot 6)$ & 0 \\
C. utilis & $1(0 \cdot 6)$ & 0 \\
C. kafyr & 0 & $1(1 \cdot 6)$ \\
C. holmii & 0 & $7(1 \cdot 6)$ \\
Unidentifiable & $1(0 \cdot 6)$ & $\mathbf{6 4}$ \\
Total & $\mathbf{1 7 3}$ & \\
\hline
\end{tabular}


has become an opportunistic pathogen causing fungaemia in immunocompromised patients (Cherifi et al., 2004; Henry et al., 2004). Three strains of S. cerevisiae were accompanied by C. albicans. The transient presence of S. cerevisiae in the oral cavity has been observed but the genetic heterogeneity of the isolates and rapid clearance suggest that the presence of $S$. cerevisiae is the result of colonization by orally adapted strains (Sweet et al., 2002). Twenty-one patients carried two species of yeasts, two patients carried three species and one patient harboured four different species of yeasts. Occurrence of multiple yeasts could not be linked to candidiasis; however, three patients who carried other yeasts with C. albicans had oral candidiasis at the time of sampling.

In addition to the above well-documented species, four nonalbicans species, Candida sake, Candida famata, Candida pelliculosa and Candida utilis, were isolated from the oral cavity of the HIV-infected subjects (Table 3). The large variety of yeasts harboured by the South African population could be attributed to life in a rural environment where there is exposure to a large variety of yeasts. The pathogenic role of these rare non-albicans yeasts in the oral cavity of HIV-positive patients needs further investigation.

\section{Effect of antifungal agents}

Another factor that could influence Candida carriage is the use of antimycotic agents. Nystatin, amphotericin B and fluconazole have been used for many years for long-term therapy in patients with HIV infection (Greenspan, 1994). The development of fluconazole resistance is an emerging trend. In 1995, $40 \%$ of patients on long-term therapy carried resistant strains, $43 \%$ in 1997 and $45 \%$ in 2000 (Magaldi et al., 2001; Tumbarello et al., 1997; Johnson et al., 1995), which threatens its clinical effectiveness. In our study, we examined the effect of previous antifungal treatment with nystatin, amphotericin B, fluconazole or miconazole on the number of viable Candida cells carried by HIVpositive patients. Overall there was no significant difference $(P=0 \cdot 05)$ between patients who had been treated previously with antimycotic drugs and those who had not been exposed (Table 4). However, 25 of the 45 patients who were treated with nystatin required further treatment while only 7 of the 37 patients treated with amphotericin $B$ required more than one treatment. Seven of the eight patients with yeast counts of greater than $10^{4}$ had received amphotericin treatment previously while one patient was treated with miconazole and fluconazole.

These findings suggest that some patients carry large numbers of yeast that are resistant to several antifungals, do not respond to treatment and are prone to candidiasis. This also suggests that the resident yeast population is stable and occupies a specific niche in the oral cavity. With treatment the level of yeast declines but is restored to the original level after treatment is discontinued. This may not apply to several non-albicans species because there are numerous reports describing the colonization and infection of compromised patients taking long-term oral antifungal agents. Primary resistance to the antifungal agent has been reported in C. krusei and C. glabrata (Wingard et al., 1991, 1993; Wingard, 1994) with C. krusei being universally resistant to fluconazole (Wingard et al., 1991). For this reason, all oral lesions with a presumptive diagnosis of candidiasis should be subjected to microscopy and culture. Isolated cultures should be identified to species level, antifungal sensitivity should be determined and primary resistance should be kept in mind during treatment.

In conclusion, this study has shown that the carrier rate of Candida is higher in South Africa than elsewhere in the world. In addition, HIV-positive patients carry more and a greater variety of yeasts than HIV-negative subjects. This may be related to several factors, including diet, oral hygiene, the number of HIV-positive patients in the population and the large number of patients who do not have access to HAART. Exposure to antifungal drugs has no effect on the level of yeast carriage in HIV-positive patients.

\section{ACKNOWLEDGEMENTS}

We thank Professor Paul Fatti for his valuable statistical advice. This research was supported by Colgate Palmolive and 3M Pharmaceutical (Pty) Ltd, South Africa.

\section{REFERENCES}

Belazi, M., Velegraki, A., Koussidou-Eremondi, T., Andreadis, D., Hini, S., Arsenis, G., Eliopoulou, C., Destouni, E. \& Antoniades, D. (2004). Oral Candida isolates in patients undergoing radiotherapy for head and neck cancer: prevalence, azole susceptibility profiles and

Table 4. Viable yeast counts in the whole saliva of HIV-positive subjects with no previous antimycotic therapy or who had antimycotic drugs previously

Values are the number of patients (\%).

\begin{tabular}{|lccr|}
\hline Study group & \multicolumn{3}{c|}{ Yeast count (c.f.u. $\mathbf{~ m l}^{\mathbf{- 1}}$ ) } \\
\cline { 2 - 4 } & $\mathbf{1 - 1 0 0 0}$ & $\mathbf{1 0 0 0 - 1 0 0 0 0}$ & $>\mathbf{1 0 0 0 0}$ \\
\hline No previous antimycotic treatment $(n=177)$ & $96(54 \cdot 2)$ & $60(33 \cdot 9)$ & $21(11 \cdot 9)$ \\
Previous antimycotic treatment $(n=78)$ & $36(46 \cdot 1)$ & $34(43 \cdot 6)$ & $8(10 \cdot 3)$ \\
\hline
\end{tabular}


response to antifungal treatment. Oral Microbiol Immunol 19, 347-351.

Blignaut, E., Raubenheimer, E., van Heerden, W. F. P., Senekal, R. \& Dreyer, M. J. (1995). Oral yeast flora of a Kalahari population. J Dent Assoc S Afr 50, 601-604.

Bodey, G., Bueltmann, B., Duguid, W. \& 11 other authors (1992). Fungal infections in cancer patients: an international autopsy survey. Eur J Clin Microbiol Infect Dis 11, 99-109.

Boerlin, P., Boerlin-Petzold, F., Durussel, C., Addo, M., Pagani, J. L., Chave, J. P. \& Bille, J. (1995). Cluster of atypical Candida isolates in a group of human immunodeficiency virus-positive drug users. J Clin Microbiol 33, 1129-1135.

Campisi, G., Pizzo, G., Milici, M. E., Mancuso, S. \& Margiotta, V. (2002). Candida carriage in the oral cavity of human immunodeficiency virus-infected subjects. Oral Surg Oral Med Oral Pathol 93, 281-286.

Cherifi, S., Robberecht, J. \& Miendje, Y. (2004). Saccharomyces cerevisiae fungemia in an elderly patient with Clostridium difficile colitis. Acta Clin Belg 59, 223-224.

Darwazeh, A. M., Al-Dosari, A. \& Al-bagieh, N. H. (2002). Oral Candida and nasal Aspergillus flora in a group of Saudi healthy dentate subjects. Int Dent J 52, 273-277.

Diamond, R. D. (1991). The growing problem of mycoses in patients infected with the human immunodeficiency virus. Rev Infect Dis 13, 480-486.

Doebbeling, B. N., Hollis, R. J., Isenberg, H. D., Wenzel, R. P. \& Pfaller, M. A. (1991). Restriction fragment analysis of a Candida tropicalis outbreak of sternal wound infections. J Clin Microbiol 29, 1268-1270.

Dorrington, R., Bradshaw, D., Johnson, L. \& Budlender, D. (2004). The Demographic Impact of HIV/AIDS in South Africa. National Indicators for 2004. Cape Town: Centre for Actuarial Research, South African Medical Research Council and Actuarial Society of South Africa.

Fidel, P. L., Jr (2006). Candida-host interactions in HIV disease: relationships in oropharyngeal candidiasis. Adv Dent Res 19, 80-84.

Greenspan, D. (1994). Treatment of oral candidiasis in HIV infection. Oral Surg Oral Med Oral Pathol 78, 211-215.

Gugnani, H. C., Becker, K., Fegeler, W., Basu, S., Chattopadhya, D., Baveja, U., Satyanarayana, S., Kalghatgi, T. \& Murlidhar, A. (2003). Oropharyngeal carriage of Candida species in HIV-infected patients in India. Mycoses 46, 299-306.

Hauman, C. H., Thompson, I. O., Theunissen, F. \& Wolfaardt, P. (1993). Oral carriage of Candida in healthy and HIV-seropositive persons. Oral Surg Oral Med Oral Pathol 76, 570-572.

Henry, S., D’Hondt, L., Andre, M., Holemans, X. \& Canon, J. L. (2004). Saccharomyces cerevisiae fungemia in a head and neck cancer patient: a case report and review of the literature. Acta Clin Belg 59, 220-222.

Hoegl, L., Thoma-Greber, E., Rocken, M. \& Korting, H. C. (1998). Persistent oral candidiasis by non-albicans Candida strains including Candida glabrata in a human immunodeficiency virus-infected patient observed over a period of 6 years. Mycoses 41, 335-338.

Jabra-Rizk, M. A., Falkler, W. A., Enwonwu, C. O., Onwujekwe, D. I., Merz, G. \& Meiller, T. F. (2001). Prevalence of yeast among children in Nigeria and the United States. Oral Microbiol Immunol 16, 383-385.

Johnson, E. M., Warnock, D. W., Luker, J., Porter, S. R. \& Scully, C. (1995). Emergence of azole drug resistance in Candida species from HIV-infected patients receiving prolonged fluconazole therapy for oral candidosis. J Antimicrob Chemother 35, 103-114.
Kadir, T., Uygun, B. \& Akyuz, S. (2005). Prevalence of Candida species in Turkish children: relationship between dietary intake and carriage. Arch Oral Biol 50, 33-37.

Knight, L. \& Fletcher, J. (1971). Growth of Candida albicans in saliva: stimulation by glucose associated with antibiotics, corticosteroids and diabetes mellitus. $J$ Infect Dis 123, 371-377.

Mackeown, J. M. \& Faber, W. M. (2004). Frequency of food items consumed by young rural and urban African children - essential knowledge to provide dietary advice in caries prevention. Int Dent $J$ 54, 284-290.

Magaldi, S., Mata, S., Hartung, C., Verde, G., Deibis, L., Roldan, Y. \& Marcano, C. (2001). In vitro susceptibility of 137 Candida sp. isolates from HIV positive patients to several antifungal drugs. Mycopathologia 149, 63-68.

Masipa, J. N., Hauman, C. H. \& Raubenheimer, E. J. (1992). Oral carriage of Candida species in patients visiting the Medunsa Dental Clinic. J Dent Assoc S Afr 47, 407-409.

Milan, E. P., Kallas, E. G., Costa, P. R., da Matta, D. A. \& Lopes Colombo, A. (2001). Oral colonization by Candida spp. among AIDS household contacts. Mycoses 44, 273-277.

Patton, L. L., Phelan, J. A., Ramos-Gomez, F. J., Nittayananta, W., Shiboski, C. H. \& Mbuguye, T. L. (2002). Prevalence and classification of HIV-associated oral lesions. Oral Dis 8 (Suppl. 2), 98-109.

Paya, C. V. (1993). Fungal infections in solid-organ transplantation. Clin Infect Dis 16, 677-688.

Pendrak, M. L., Yan, S. S. \& Roberts, D. D. (2004). Sensing the host environment: recognition of hemoglobin by the pathogenic yeast Candida albicans. Arch Biochem Biophys 426, 148-156.

Pfaller, M. A. (1996). Nosocomial candidiasis: emerging species, reservoirs, and modes of transmission. Clin Infect Dis 22 (Suppl. 2), S89-S94.

Pinjon, E., Sullivan, D., Salkin, I., Shanley, D. \& Coleman, D. (1998). Simple, inexpensive, reliable method for differentiation of Candida dubliniensis from Candida albicans. J Clin Microbiol 36, 2093-2095.

Pizzo, G., Giuliana, G., Milici, M. E. \& Giangreco, R. (2000). Effect of dietary carbohydrates on the in vitro epithelial adhesion of Candida albicans, Candida tropicalis and Candida krusei. New Microbiol 23, 63-71.

Pongsiriwet, S., lamaroon, A., Sriburee, P., Pattanaporn, K. \& Krisanaprakornkit, S. (2004). Oral colonization of Candida species in perinatally-HIV infected children in northern Thailand. J Oral Sci 46, 101-105.

Powderly, W. G. (1992). Mucosal candidiasis caused by non-albicans species of Candida in HIV-positive patients. AIDS 6, 604-605.

Price, M. F., LaRocco, M. T. \& Gentry, L. O. (1994). Fluconazole susceptibilities of Candida species and distribution of species recovered from blood cultures over a 5-year period. Antimicrob Agents Chemother 38, 1422-1424.

Qi, Q. G., Hu, T. \& Zhou, X. D. (2005). Frequency, species and molecular characterization of oral Candida in hosts of different age in China. J Oral Pathol Med 34, 352-356.

Redding, S. W., Dahiya, M. C., Kirkpatrick, W. R., Coco, B. J., Patterson, T. F., Fothergill, A. W., Rinaldi, M. G. \& Thomas, C. R., Jr (2004a). Candida glabrata is an emerging cause of oropharyngeal candidiasis in patients receiving radiation for head and neck cancer. Oral Surg Oral Med Oral Pathol Oral Radiol Endod 97, 47-52.

Redding, S. W., Marr, K. A., Kirkpatrick, W. R., Coco, B. J. \& Patterson, T. F. (2004b). Candida glabrata sepsis secondary to oral colonization in bone marrow transplantation. Med Mycol 42, $479-481$. 
Reichart, P. A., Samaranayake, L. P., Samaranayake, Y. H., Grote, M., Pow, E. \& Cheung, B. (2002). High oral prevalence of Candida krusei in leprosy patients in northern Thailand. J Clin Microbiol 40, 44794485.

Roilides, E., Farmaki, E., Evdoridou, J. \& 7 other authors (2003). Candida tropicalis in a neonatal intensive care unit: epidemiologic and molecular analysis of an outbreak of infection with an uncommon neonatal pathogen. J Clin Microbiol 41, 735-741.

Samaranayake, L. P., Hughes, A. \& MacFarlane, T. W. (1984). The proteolytic potential of Candida albicans in human saliva supplemented with glucose. J Med Microbiol 17, 13-22.

Sanchez, V., Vazquez, J. A., Barth-Jones, D., Dembry, L., Sobel, J. D. \& Zervos, M. J. (1993). Nosocomial acquisition of Candida parapsilosis: an epidemiologic study. Am J Med 94, 577-582.

Schmidt-Westhausen, A., Schiller, R. A., Pohle, H. D. \& Reichart, P. A. (1991). Oral Candida and Enterobacteriaceae in HIV-1 infection: correlation with clinical candidiasis and antimycotic therapy. J Oral Pathol Med 20, 469-472.

Staib, P. \& Morschhauser, J. (1999). Chlamydospore formation on Staib agar as a species-specific characteristic of Candida dubliniensis. Mycoses 42, 521-524.

Sweet, S. P., Howell, S. A., Abebe, B. \& Naglik, J. R. (2002). Oral colonization by Saccharomyces cerevisiae. Oral Dis 8 (Suppl. 2), 185.
Teanpaisan, R. \& Nittayananta, W. (1998). Prevalence of Candida species in AIDS patients and HIV-free subjects in Thailand. $J$ Oral Pathol Med 27, 4-7.

Torres, S. R., Peixoto, C. B., Caldas, D. M., Silva, E. B., Magalhaes, F. A., Uzeda, M. \& Nucci, M. (2003). Clinical aspects of Candida species carriage in saliva of xerostomic subjects. Med Mycol 41, 411-415.

Tsang, C. S. \& Samaranayake, L. P. (2000). Oral yeasts and coliforms in HIV-infected individuals in Hong Kong. Mycoses 43, 303-308.

Tumbarello, M., Tacconelli, E., Caldarola, G., Morace, G., Cauda, R. \& Ortona, L. (1997). Fluconazole resistant oral candidiasis in HIVinfected patient. Oral Dis 3 (Suppl. 1), S110-S112.

Wingard, J. R. (1994). Infections due to resistant Candida species in patients with cancer who are receiving chemotherapy. Clin Infect Dis 19 (Suppl. 1), S49-S53.

Wingard, J. R., Merz, W. G., Rinaldi, M. G., Johnson, T. R., Karp, J. E. \& Saral, R. (1991). Increase in Candida krusei infection among patients with bone marrow transplantation and neutropenia treated prophylactically with fluconazole. N Engl J Med 325, 1274-1277.

Wingard, J. R., Merz, W. G., Rinaldi, M. G., Miller, C. B., Karp, J. E. \& Saral, R. (1993). Association of Torulopsis glabrata infections with fluconazole prophylaxis in neutropenic bone marrow transplant patients. Antimicrob Agents Chemother 37, 1847-1849. 